\title{
Parallel Simulation of Laser Propagation Based on Component Modeling
}

\author{
Xie Xiaogang, Zhang Jianzhu, Yue Yufang, An Jianzhu, Zhang Feizhou \\ Institute of Applied Physics and Computational Mathematics, Beijing 100094, China \\ tom_xxg@126.com
}

Keywords: laser propagation simulation; parallel computing; component modeling; load balancing; MPI

\begin{abstract}
Due to the large computation quantity of laser propagation simulation in complicated laser systems, the traditional serial simulation cannot fulfill the requirement. In this paper, the laser propagation algorithms of coherent light source and noncoherent light source were introduced, two parallel computing modes (parallelization among multi beams and parallelization in one noncoherent beam) were proposed, and a type of data allocation algorithm for load balancing was designed. These were all implemented in EasyLaser which is the component-based laser system simulation software built by us. It has the abilities of fast building parallel simulation programs and can reduce the difficulty to develop parallel programs. In the final, some performance tests for parallel computing were performed on a typical laser propagation simulation model. The test results indicate that the applying of the parallel algorithms can effectively take advantage of the hardware resources and reduce the time consuming.
\end{abstract}

\section{Introduction}

Along with the increase of the complexity of laser systems, people often need to do the synchronous simulation of the propagation of multi-beams (including the coherent beams and the noncoherent beams). In order to promote the precision, the scale of the computing mesh is continually increasing. And as the increase of the distance of the lasers propagation path and the increase of the number of the optical components which are passed by the lasers, the computation quantity is greatly increased. The traditional method of serial simulation has the problems of long-time comsuming and low efficiency, which are not good to do the studies. On the other hand, CPU's technology has entered the stage of multi-core, and is developping to the stage of many-core [1]. Therefore, the parallelization of the laser propagation simulation is a good idea to promote the speed of the simulation and the use ratio of the hardware resources [2, 3].

The general tools for implementing the parallel programs are MPI [4] and OpenMP [5]. Many current researches make use of the APIs provided by MPI and OpenMP to develop the parallel computing programs for the simulation of the laser propagation. For example, Ma Cunliang et al [6] implemented the parallel simulation of femtosecond laser pulses propagation in air based on OpenMP. The direct using of the APIs requires the researchers not only know the knowledge of the optics, but also be accomplished in the complicated technique of the parallel programing. This method has the problems of hard to develop, low effeciency, hard to do secondary modeling, high coupling degree and hard to coordinate within models, which result in the difficulties to implement the efficient developping, encapsulation and fast assembly of models.

In order to enhance the simulation speed of complicated models, and to reduce the difficulty to develop the parallel programs, which can make the researchers be absorbed in the analysis of physical problems, we designed and implemented the components library which supports multi-process parallel simulation in EasyLaser [7] which is a component-based laser system simulation software built by us. As a result, reseachers can fast and neatly build various models of laser propagation on EasyLaser by simple and intuitionistic modeling operations. In this way, they can easily implement the parallel simulation of laser propagation without complicated parallel programing. This method can effectively take advantage of the capabilities of the hardware resources of multi-core and many-core, which can reduce the time consuming for the simulation on EasyLaser and expand the 
applying areas of EasyLaser, and can also promote the efficiency of the modeling of the parallel simulation for complicated laser systems, and reduce the difficulty to develop parallel programs.

\section{Introduction to the Simulation Algorithms of Laser Propagation}

Due to the feature, the light sources have the types of coherent source and noncoherent source.

The plane wave light source and the point light source are generally considered as the coherent sources. The propagation of the coherent source can be depicted by Maxwell equations. The propagation of the single color electromagnetic wave with polarization in the medium without free charges, free electrical currents and magnetism can be depicted by the following paraxial approximation formula:

$$
2 i k \frac{\partial \psi}{\partial z}+\nabla_{\perp}^{2} \psi+k^{2} \delta \varepsilon \psi=0
$$

In Eq. $1, \nabla_{\perp}^{2}=\partial^{2} / \partial x^{2}+\partial^{2} / \partial y^{2}, \delta \varepsilon=\delta \varepsilon_{T B}+\delta \varepsilon_{T u r b} . \delta \varepsilon_{T B}, \delta \varepsilon_{\text {Turb }}$ are the variatations of the atmosphere refractive index produced by thermal blooming and turbulance. $\mathrm{k}=2 \pi / \lambda$ ( $\lambda$ is the wavelength). $\psi$ is the amplitude of the light field.

The variatations of density and temperature can be depicted by hydrodynamic equations with isobar approximation:

$$
\begin{aligned}
& \frac{\partial \rho_{1}}{\partial t}+\vec{V} \cdot \nabla_{\perp} \rho_{1}=-\frac{\gamma-1}{C_{s}^{2}} \alpha_{a} I_{p} . \\
& \frac{\partial T_{1}}{\partial t}+\vec{V} \cdot \nabla_{\perp} T_{1}=\frac{\alpha_{a}}{\rho_{0} C_{p}} I_{p} .
\end{aligned}
$$

In the above equations, ${ }^{I}=\left(\varepsilon_{0} c / 2\right) \psi \psi * \exp \left[-\int_{0}^{Z} \alpha_{t}\left(z^{\prime}\right) d z^{\prime}\right], V$ is the wind speed perpendicular to the direction of propagation, $\alpha_{t}$ is the extinction coefficient, $\alpha_{a}$ is the absorption coefficient, $\gamma=1.4$ is the adiabatic coefficient, $C_{S}=340 \mathrm{~m} / \mathrm{s}$ is the sound speed, $C_{P}=1.006 \mathrm{~J} / \mathrm{g}$ is the isobaric specific heat capacity. Simulation of the laser propagation in atmosphere can be done by solving the simultaneous Eq. 1 - Eq. 3.

The process of the laser propagation in atmosphere can be approximately depicted by Fig. 1 . The whole light path is devided into $\mathrm{N}$ phase screens, which represent the wavefront aberration phases produced by the turbulance and thermal blooming. The propagations between any two phase screens are considered as the vacuum propagation.

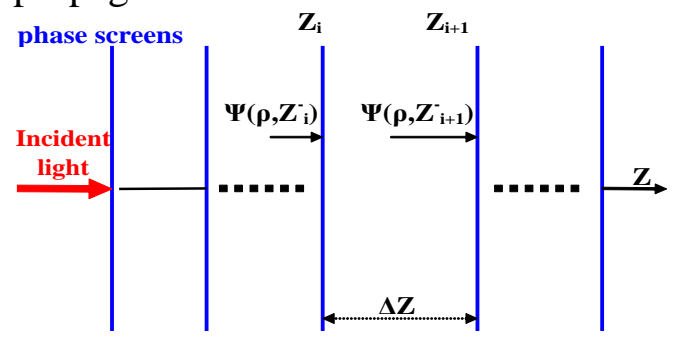

Figure 1 . The sketch of the model of the laser propagation in atmosphere

The propagations of laser beams among mirrors can be considered as the vacuum propagation, while the compositive effect of the mirrors' connatural aberrations and the aberrations produced by the thermal deformation on the mirrors and the medium can be considered as a phase screen. When we study on the laser propagation with the theory of wave optics, we should also consider the effects of geometry optics, such as the beam reflection and spectroscopy.

The artificial beacons (Rayleigh and $\mathrm{Na}$ ) and the diffuse reflection of the extended target can be considered as noncoherent sources. The target source light should be propagated back to the sensor through the atmosphere to do the simulation of the imaging of one extended target [8]. The method is descripted as follow. First divide the source image into a matrix of small pieces within isoplanatic 
angle of the turbulance, the pixels in the same piece have the same point spread function, different pieces have different point spread functions; Then do propagation for each piece using the method depicted in Fig. 1, and do convolution with single piece and the point spread function to get the image of the piece; Finally do nocoherent superposition with all the images of the pieces to get the whole image of the extended target after the propagation in atmosphere (Fig. 2).

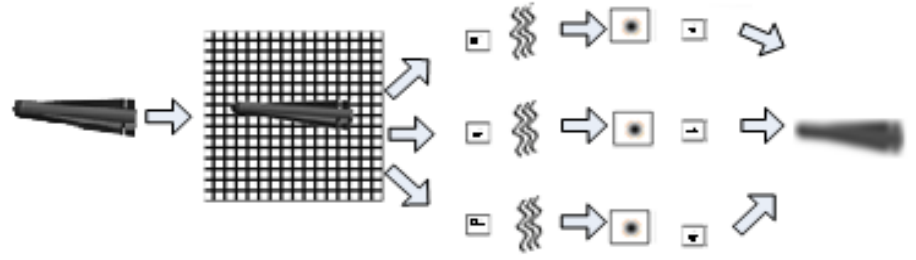

Figure 2. The sketch of the algorithm of the noncoherent beam propagation in atmosphere

\section{Designs of the Parallel Modes}

\subsection{Parallelization among Multi Beams}

The laser beam may pass many optical mirrors and the atmosphere on the path of the propagation, each segment can be simulated by the algorithm introduced in part 2 . There is strict data dependence among these segments, which means that the latter process can not start until the former process have been finished, so these segments are not suitable for parallel computing and should be executed by single computing process. While the propagations of different beams are independent, this means that there is no data dependence among them, so they are quite suitable for parallel computing and can be executed by different computing processes.

When different beams pass the same medium, the affection to the propagation of each beam produced by the change of the medium's property should also be considered. For example, when the deformable mirror's surface or the atmosphere's phase screens are changed, the propagation of each beam should be affected. The solution in parallel computing is that: after each iteration step, the new surface or phase screens are updated to every computing process by MPI messages, and will be used in the next iteration step.

\subsection{Parallelization in One Noncoherent Beam}

In the propagation algorithm of noncoherent beam introduced in part 2, the atmosphere propagations of every piece do not affect each other, which means that they have typical data independence, so they can be executed by different computing processes. For the simulation of the imaging of the extended targets, a large number of pieces are usually divided, which is the keystone of parallelization. The following algorithm depicts how to balancedly allocate $n \times n$ pieces to $m$ computing processes.

Suppose there is one noncoherent beam which contains $n \times n$ pieces $\left(B_{0}, B_{1}, \ldots, B_{n \times n-1}\right)$, each piece has a point spread function, and all pieces are numbered from left to right in row first sequence (Fig. 3(a)), the dimensions of every point spread function are the same. We designed the following algorithm to allocate all the pieces to $m$ computing processes $\left(P_{0}, P_{1}, \ldots, P_{m-1}\right)$.

The pieces set $S_{i}$ for process $P_{i}(i=0,1,2, \ldots, m-1)$ is caculated by Eq. 4, where $b=\left\lfloor n^{2} / m\right\rfloor$, $k=\bmod \left(n^{2}, m\right)$.

$$
S_{i}= \begin{cases}\left\{B_{i \times b+i}, B_{i \times b+i+1}, \ldots, B_{i \times b+i+b}\right\} & ,(0 \leq i<k) \\ \left\{B_{i \times b+k}, B_{i \times b+k+1}, \ldots, B_{i \times b+k+b-1}\right\} & ,(k \leq i \leq m-1)\end{cases}
$$

If $k=0$, all the pieces are equally allocated to each process, which contains $b$ pieces, $S i=\left\{B_{i \times b}\right.$, $\left.B_{i \times b+1}, \ldots, B_{i \times b+b-1}\right\}(i=0,1, \ldots, m-1)$; if $k>0$, each of the first $k$ processes contains $b+1$ pieces, each of the last $m-k$ processes contains $b$ pieces. This algorithm insures that the numbers of the pieces allocated to each process are closest, and the discrepancy number is max to 1 , so the load of each process is almost balanced. The result of allocating $3 \times 3$ pieces to 4 processes is shown in Fig. 3(b). 


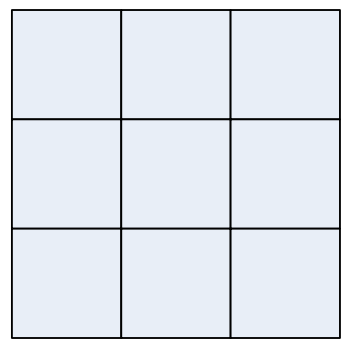

(a) $3 \times 3$ pieces

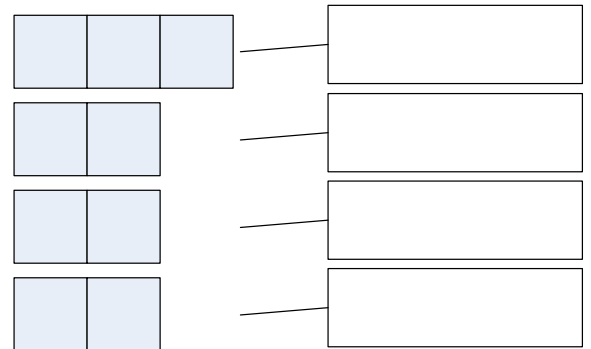

(b) The distributing of $3 \times 3$ pieces to 4 processes

Figure 3 . The sketch of the pieces allocation algorithm in noncoherent beam

When the noncoherent beams arrive at the sensor for imaging, nocoherent superposition should be done with all the images of each piece to form a whole image, this is a serial process. At the time, main process $P_{0}$ shoud receive all the other images of pieces from other processes by MPI messages, and compose them to form the whole image.

\section{The Parallel Simulation Implemented in EasyLaser}

EasyLaser is the component-based laser system simulation software built by us. It was built on the base of many years' mature simulation modules. It is composed of the general purpose component-based model and simulation platform called SciSimu and 8 libraries of modules which have the ablities to model and simulation the laser propagations among optical mirrors, the propagation in atmosphere, sensor imaging, Adaptive Optics (AO) and target tracing. The most remarkable feature of EasyLaser is the capability of fast building different laser system models and doing simulation analysis by simple operations.

Currently the modules simulating the coherent sources are "plane wave source" and "point source"; the modules simulating the noncoherent sources are "scene source", "extended target" and "attificial beacon"; the modules having the ability of laser propagation are "deformable mirror", "steering mirror”, "telescope”, "reflector mirror”, "splitter mirror”, “window mirror”, and "atmosphere path”; the modules having the ability of imaging are "Hartmann sensor" and "TV sensor".

The two parallel computing modes mentioned above (parallelization among multi beams and parallelization in one noncoherent beam) have been implemented in EasyLaser, and can be simultaneously used in one time simulation. A model of laser propagation built on EasyLaser is shown in Fig. 4, it contains two light sources: the plane wave source depicted by red lines and the exptend target source depicted by green lines. The plane wave laser beam propagates pass a serial of mirrors and the atmosphere to the target board. While the extended target beam propagates along the reverse path to Hartmann sensor for AO correction.

The config dialog box for simulation run is shown in Fig. 5. As a matter of convenience, automatic recognization of different beams from the block diagram (Fig. 4) is done for the users to set the total number of the computing processes and the allocation of which beam will be executed in which process. As there is no fussy programing and debugging from the modeling to the simulation run, the working efficiency is greatly promoted by EasyLaser.

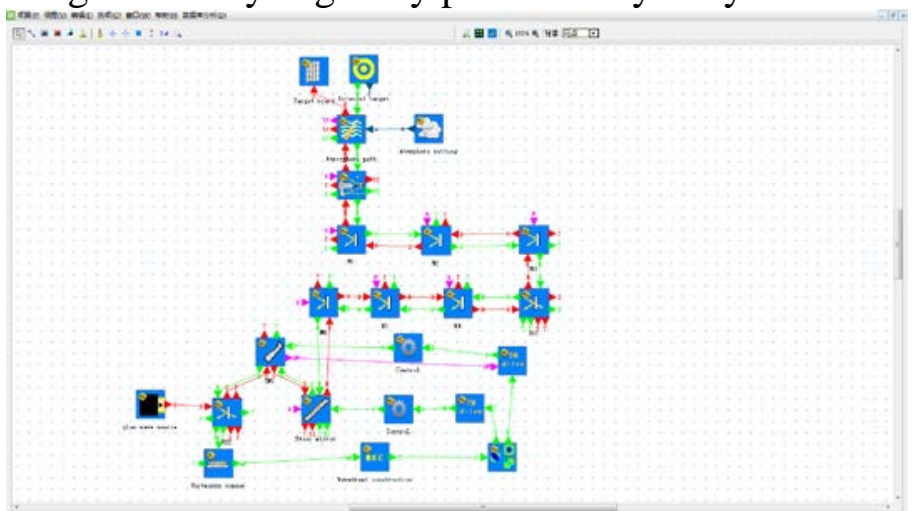

Figure 4. A model of laser propagation on EasyLaser

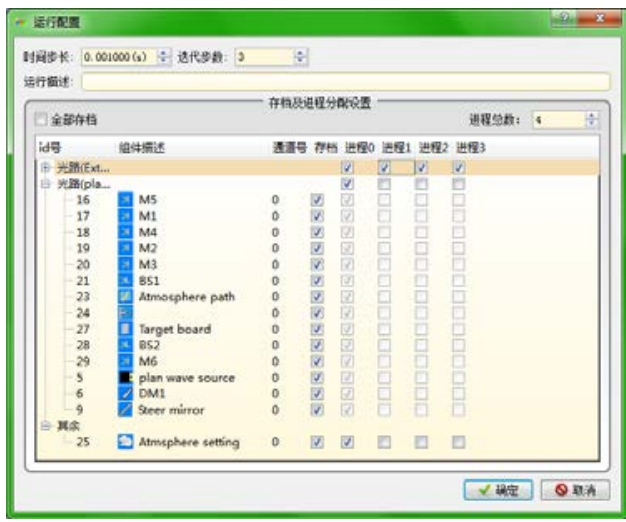

Figure 5. Allocation of processes 


\section{The Analysis of the Parallel Performance}

The parallel performance is related to the specific model and the allocation of computing processes. Figure 4 is a typical model of the laser propagation, which was choosed as the object to do the tests. In this model, the dimension of the mesh for the plane wave source beam is $512 \times 512$, the number of the small pieces divided for the extended target beam is $512 \times 512$ and the dimension of the mesh for each piece is $128 \times 128$. It is not difficult to discover that the computation quantity of the plane wave beam is equal to the computation quantity of 16 pieces in the extended target beam and the most computation quantity is on the propagation of the extended beam. So we allocated the extended beam's small pieces to multi processes and the plane wave beam to single process.

The parallel performance tests were done on IBM cluster which has 11 nodes, each node has 2 CPUs, and each CPU has 4 cores. We respectively do the simulation tests with 1 process, 17 processes, 33 processes and 65 processes, each test executes 20 iteration steps. The time consuming, speedup and parallel efficiency are listed in Table 1. It shows that: the parallelization can greatly promote the speed of simulation and shorten the time consuming; by properly increase the number of processes according to the computer's CPU cores and the feature of the model, the time consuming can be shorter; the increase of the number of the processes causes the speedup grows up but the parallel efficiency goes down a little which is caused by the increase of the messages and some serial computing modules (such as the initial of the matrix of wavefront decomposition) which may make the spending for the synchronization among computing processes.

Table 1. Test results of parallel performance

\begin{tabular}{cccccc}
\hline \multirow{2}{*}{$\begin{array}{c}\text { Number of } \\
\text { processes }\end{array}$} & \multicolumn{2}{c}{ Process id allocated to beams } & Time (s) & Speedup & $\begin{array}{c}\text { Parallel } \\
\text { efficiency }\end{array}$ \\
\cline { 2 - 3 } & Plan wave & $\begin{array}{c}\text { Extended } \\
\text { beam }\end{array}$ & & & \\
\hline 1 & 0 & $1-16$ & 3252.75 & 15.57 & $91.6 \%$ \\
17 & 0 & $1-32$ & 1769.58 & 28.62 & $86.7 \%$ \\
65 & 0 & $1-64$ & 1031.89 & 49.08 & $75.5 \%$ \\
\hline
\end{tabular}

In order to further promote the speedup and the parallel efficiency, more finely granular parallel computing will be applied to more modules, and the parallelization of iteration steps on pipeline will be introduced in the future.

\section{Conclusion}

Two parallel computing modes including parallelization among multi beams and parallelization in one noncoherent beam were designed and implemented in EasyLaser which is the component-based laser system simulation software built by us. Reseachers can easily implement the parallel simulation of laser propagation on EasyLaser by simple and intuitionistic modeling operations without complicated parallel programing, which greatly reduces the difficulty to develop parallel programs. Some performance tests for the parallel computing were performed on a typical laser propagation simulation model, and the results indicate that the applying of the parallel algorithm can effectively take advantage of the hardware resources and can reduce the time consuming.

\section{References}

[1] Manferdelli J L, Govindaraju N K, Crall C. Challenges and opportunities in many-core computing [J]. Proceedings of the IEEE, 2008, 96(5):808-815.

[2] Su Nianle, Hou Hongtao, Yang Feng, et al. Optimistic parallel discrete event simulation based on multi-core platform and its performance analysis [C]. International Conference on Complex, Intelligent and Software Intensive Systems, 2009: 675-680. 
[3] Wang Kun, Zhang Yu, Wang Huayong. Parallelization of IBM Mambo System simulator in functional modes [J]. ACM SIGOPS Operating Systems Review, 2008, 42(1): 71-76.

[4] Tang Long, He Bing, Zhang Wu. Application and research on three-dimensional electromagnetic radiation calculation using MPI+OpenMP paradigms [J]. MICROELECTRONICS \& COMPUTER, 2014, 31(8): 127-131.

[5] Luo qiuming, Ming zhong, Liu gang, et al. OpenMP compiler theory and implementation techniques [M]. Beijing: Tsinghua University, 2012.

[6] Ma Cunliang, Jia Mingzhen, Lin Wenbin. Parallel simulation of femtosecond laser pulses propagation in air based on OpenMP [J]. HIGH POWER LASER AND PARTICLE BEAMS, 2015, 27(11): 111002.

[7] Xie Xiaogang, Yue Yufang, Zhang Jianzhu, et al. EasyLaser: component-based laser system simulation software [J]. HIGH POWER LASER AND PARTICLE BEAMS, 2013, 25(10): 2536-2540.

[8] Yue Yufang, Xie Xiaogang, Zhang Jianzhu, et al. Beam control system component simulation and separation method of kinematic coupling [J]. HIGH POWER LASER AND PARTICLE BEAMS, 2014, 26(9): 091006. 